\title{
Personalising the evaluation of substance misuse treatment: a new approach to outcome measurement
}

Paula C. G. Alves, Célia M. D. Sales \& Mark Ashworth

INTERNATIONAL JOURNAL OF DRUG POLICY - IN PRESS

Patient involvement in healthcare, in general, and in substance misuse in particular, has become a topic of paramount importance (Rutter et al., 2004). Patient involvement can be conceptualised as listening to the patients' perspective and encouraging patients to take an active role in the care they are receiving. This approach is advocated by international authorities in health and social care such as the United Kingdom's NICE, which recommends "personcentred care" that takes into account the patient's "needs, preferences and strengths" (Crawford, 2011). According to Orford (2008), the perspectives of patients in substance misuse treatment tend to be overlooked and their involvement with treatment is limited. However, the evidence on how patient involvement can be improved within the context of substance misuse programmes are scarce. Can we do something to shift this reality?

In healthcare, patients can become actively involved by collaborating with treatment outcome evaluation. In psychological treatments the commonest approach to this involves the use of standardised outcome measures (SOM), whose pre-set items, developed by research experts or professionals are rated by patients. The majority of SOM, both in psychological treatments (e.g., CORE-OM; Evans et al., 2000) and in substance misuse (e.g., TOP; Marsden et al., 2008), have had their psychometric properties extensively explored and their results are widely acknowledged as valid and reliable to evaluate treatment. However, due to their universal scope and applicability to both healthy populations and those with mental health conditions, SOM are unlikely to cover all the subjective concerns of patients, their priorities and their personal experiences in treatment.

There is a different approach to evaluate treatment, which is based on patientgenerated, or individualised, outcome measures (PGOM). PGOM include items that are created by patients, resulting in personalised tailor-made scales that can be rated for severity, just like 
SOM (Sales \& Alves, 2012). There are several protocols to generate these personalised scales: for instance, the Personal Questionnaire (PQ; Elliott, Mack \& Shapiro, 1999) is a semistructured interview where patients are asked to say which are "the main problems that led them to treatment" and then to rank order them by importance; or PSYCHLOPS (Ashworth et al., 2004), a self-report instrument where patients write down the two main problems they are currently experiencing and one thing that has become difficult to do because of those problems. When PGOM are used, the level of patient involvement with treatment evaluation increases substantially, which is important not only because patients and clinicians tend to appraise treatment differently, but also because most measurements are developed without direct input from patients (Crawford et al., 2011). Also, PGOM data is not fixed in time and allows patients to revise, delete obsolete problems or add new ones that might have arisen during therapy.

PGOM are becoming increasingly popular in psychological treatments, given their advantages for outcome evaluation and clinical practice. As outcome measures, PGOM include patient case-specific information, as well as scores that quantify the distress caused by such problems, allowing a personalised evaluation of outcome (Sales \& Alves, 2012); one of the properties of PGOM is greater sensitivity were found to be more sensitive to clinical change when compared with SOM; the trade-off is that PGOM have slightly lower levels of test-restest reliability and internal reliability (Lacasse et al, 1999). Nevertheless, the reliability levels of PGOM are satisfactory and this should not detract from their role as highly sensitive measures of change (Ashworth et al., 2008; Elliott et al., 2014); PGOM allow outcome measurement to take patient's perspectives into account, making them feel more valued as individuals (Alves, Sales \& Santos, 2014). As clinical tools, PGOM have been considered to be useful to complement diagnoses, case formulation and clinical-decision making (Sales et al., 2007; Sales, Alves, Evans \& Elliott, 2014); and recently, patients reported satisfaction with the freedom that PGOM give them to focus on the topics which they prioritise during the evaluation process (Alves, Sales \& Santos, 2014).

But there are downsides to using PGOM. For instance, they have been considered time-consuming and are difficult to use with patients with certain impaired cognitive functions (e.g. memory). Moreover, PGOM hinder the comparison between patients (Sales et al., 2007; Soares et al., 2012), making it difficult to interpret their scores against population norms since 
each 'individualised' measure is, in effect, offering a score for unique, individually defined items Hence, PGOM are not the "holy grail" of outcome assessment and cannot provide, alone, all the information that is needed to evaluate patients' clinical situation.

It has been recently suggested that PGOM should be added to standardised data for optimal results, in a strategy called personalised outcome measurement approach (Sales \& Alves, 2012). In practice, this approach implies the combination of PGOM and SOM in the same evaluation protocol. Here is one example: to use, at the pre-treatment evaluation session, $P Q$ or PSYCHLOPS, followed by CORE-OM. With SOM, therapists can quickly assess the patient's level of distress based on clinical cut-offs; whilst PGOM identify the specific concerns of patients, giving more insight about how they perceive their own clinical situation. According to the characteristics of the service, personalised outcome protocols can be administered pre and post treatment; or on a session-to-session basis, to monitor the progress of patients throughout treatment. In such cases, as aforementioned, patients are able to update the contents contained in their PGOM, as many times as desired.

The personalised outcome measurement approach has already been implemented in various mental health settings, from university-based counselling centres to day psychiatry units and also group therapy interventions, with positive results. These studies have been conducted as part of a practice-based research network, dedicated to personalised assessment, the IPHA Group (Sales, Alves, Evans \& Elliott, 2014).

As earlier stated, failing to include the patient's perspective has been identified as one of ten areas which require a paradigm shift in psychological addiction treatment (Orford, 2008). If the personalised outcome measurement approach seems to work, on the one hand, and is, on the other, a potential strategy to increase patients' involvement in treatment, could we extend it to this specific context? What would we gain from doing so?

In substance misuse, improvements in mental health are among the most important treatment goals (Wanigaratne et al., 2005). However, psychological health tends to be evaluated with SOM such as the SF Health Survey (see Drug Treatment Outcomes Research Study, Jones et al., 2009 for an example), where the level of patient involvement is minimal, as previously explained. Also, as shown in previous reviews (e.g. Livingston et al., 2011), substance misuse disorders tend to be more stigmatized in comparison with other health 
problems. This may not only act as a barrier for seeking healthcare, but also to poor and inadequate provision of care, potentially leading to early treatment drop-out and poor adherence (Livingston et al., 2011).

From a clinical perspective, we believe that substance misuse treatment services could benefit from adopting the personalised measurement approach. First, the use of PGOM potentially allows clinicians to better understand how patients perceive their own situation. This is particularly relevant at the pre-treatment stage, because it helps clinicians preparing treatment plans that take the priorities of patients into account, instead of adopting "by-thebook", or protocol driven interventions. Also, in a population where drop-out rates are relatively high, to formally ask for patients' help to evaluate their own situation might increase their motivation to continue with the treatment programme.

Second, adding PGOM to outcome measurement encourages patients to select and prioritise their own problems, regardless of whether these are drug-related or not. In this context, the tools used for screening and outcome measurement purposes focus primarily on drug use and drug-related risk behaviours (e.g. TOP; Marsden et al., 2008). However, patients may have other concerns that are perceived as of equal or greater importance than their drug use (e.g. recent death of a relative). As a female patient put it in focus group recently conducted in Portugal, "This questionnaire [PSYCHLOPS, a PGOM] helps people to think about all their difficulties in life. It is not just the alcohol' (Alves, Sales \& Santos, 2014). Thus, even though PGOM do not substitute for diagnostic interviews or disorder-specific scales (e.g. Beck Depression Inventory) they may serve as flag alerts for clinical practice and further assessments.

We also hope that using PGOM will contribute to diminishing, to some extent, the stigmatization of patients with substance misuse problems. It is not uncommon for these patients to be perceived as manipulative and untruthful, which may be related to reasons why their own perspectives tend to be overlooked. However, as illustrated in the previous paragraph, asking patients' opinions about their own problem priorities enables health care workers to offer more holistic care (cf. above: its not just the alcohol)

The personalised outcome assessment approach is also a potential tool to influence policies regarding treatment provision in this field. On the one hand, this measurement 
approach provides an action plan, ultimately in the format of guidelines, for therapists to understand how to put the principle of "patient-centered" care into practice, as a means of improving quality of care in general. This is of importance at the time being, when health care budgets are increasingly constrained and funding decisions are dependent upon evidence of effectiveness judged both by professionals and patients.

Second, personalised outcome measurement is a strategy that reconciles the existing approach to outcome assessment, with personalised questionnaires that serve not only as complementary outcome measures, but also as clinical tools that are useful to practice, and humanize the assessment procedure that is often regarded as impersonal 'tick-box' measures.

As PGOM reflect the patient's perspectives, we believe that they might provide insights about the problems which are most relevant to this population, so that we know, through the patient's eyes, which areas should be more emphasized in drug misuse treatment programs. For instance, if the majority of patients report that unemployment is what concerns them the most, it might be necessary to revise the policies regarding social rehabilitation of patients in drug misuse treatment (e.g. increasing the number of partnerships between treatment centres and employment agencies).

Also, adding PGOM to traditional outcome measures will lead to more comprehensive policies of treatment evaluation and delivery, for it includes the point of view of all the parties involved in healthcare, namely, the voice of patients who are likely to be unheard in substance misuse settings.

Finally, as aforementioned, the use of tools to include the perspective of patients will, ultimately, contribute to a greater humanization of the substance misuse treatment system. All in all, from an ethical point of view, there is an imperative to enhance patient autonomy in patient care. By valuing what patients have to say - and involving them as actively as possible in the delivery of health care, we consider that this approach will maximize the potential benefit of treatment programmes.

There are, however, several steps that need to be taken before this approach is fully acknowledged as feasible and reliable in substance misuse treatment. These include: 1) Piloting the personalised assessment approach in several international treatment agencies, to increase its robustness; 2) Discussing, with international drug monitoring agencies, the 
appropriateness of developing and disseminating international guidelines for personalised assessment, to reduce the gap between treatment delivery and outcome assessment across countries; 3) Creating practice-based networks for drug treatment services to collaborate and share their experiences with personalised assessment, extending the cooperation between therapists, researchers and patients towards the improvement of healthcare (e.g. International Exchange Platform for Personalising Substance Misuse Treatment; Alves, Sales \& Ashworth, 2013).

To sum up, in this paper we have presented the personalised outcome measurement approach as a potential strategy to increase patient involvement with substance misuse treatment, an area in need of an urgent paradigm shift. Overall, this strategy potentially allows us to better accommodate the diversity and the idiosyncrasies of each patient that enters each consultation room. There is, however, the danger of becoming so individualized that it becomes difficult to have an overview about the population's heterogeneity, rendering it harder to plan services at a population level. Hopefully, the compromise that we propose between personalised and standardised measures is the ideal strategy to bridge these two worlds, but only further testing of where the balance lies will enable us to maximize the benefit from both approaches.

\section{References}

Alves, P. C. G., Sales, C. M. D., \& Santos, M. T. (2014, June). Involving patients in healthcare provision [PowerPoint slides]. Paper presented at the International Meeting of the Society for Psychotherapy Research, Copenhagen, Denmark.

Alves, P., Sales, C., \& Ashworth, M. (2013). Enhancing the patient involvement in outcomes: a study protocol of personalised outcome measurement in the treatment of substance misuse. BMC Psychiatry, 13, 337-343. 
Ashworth, M., Shepherd, M., Christey, J., Matthews, V., Wright, K., \& Parmentier (2004). A client-generated psychometric instrument: the development of "PSYCHLOPS."Counselling and Psychotherapy Research, 4(2), 27-31.

Ashworth, M., Evans, C., \& Clement, S. (2008). Measuring psychological outcomes after cognitive behaviour therapy in primary care: a comparison between a new patient-generated measure "PSYCHLOPS" (Psychological Outcome Profiles) and "HADS" (Hospital Anxiety and Depression Scale). Journal of Mental Health, 18(2), 169-177.

Crawford M (chair) (2011). Service user experience in adult mental health: improving the experience of care for people using adult NHS mental health services. Manchester: National Health Service.

Crawford, M. J., Robotham, D., Thana, L., Patterson, S., Weaver, T., \& Barber, R. (2011). Selecting outcome measures in mental health: the views of service users. Journal of Mental Health, 20(4), 336-346.

Elliott, R. et al. Psychometrics of the Personal Questionnaire: A Client-Generated Outcome Measure. Manuscript under revision.

Elliott, R., Mack, C., \& Shapiro, D. A. (1999). Simplified Personal Questionnaire Procedure. Toledo: University of Toledo.

Evans, C., Connell, J., Barkham, M., Mellor-Clark, J., \& Audin, K. (2000). Towards a standardised brief outcome measure: psychometric properties and utility of the CORE-OM. The British Journal of Psychiatry, 180, 51-60.

Jones, A., Donmall, M., Millar, T., Moody, A., Weston, S., \& Anderson, T. (2009). The Drug Treatment Outcomes Research Study (DTORS): Final outcomes report. United Kingdom. 
Marsden, J., Farrell, M., Bradbury, C., Dale-Perera, A., Eastwood, B., Roxburgh, M., \& Taylor, S. (2008). Development of the Treatment Outcomes Profile. Addiction, 443, 1450-60.

Orford, J. (2008). Asking the right questions in the right way: the need for a shift in research on psychological. Addiction, 103(6), 875-885.

Rutter, D., Manley, C. Weaver, T., Crawford, M. J., Fulop, N. (2004). Patients or partners? Case studies of user involvement in the planning and delivery of adult mental health services in London. Social Science \& Medicine, 58, 1973-1984.

Sales, C. M. D., Alves, P. C. G., Evans, C., \& Elliott, R. (2014). The Individualized Patient Progress System: A decade of collaborative networking. Counselling \& Psychotherapy Research, 14, 181-191.

Sales, C. M. D., \& Alves, P. C. G. (2012). Individualized Patient-Progress Systems: Why We Need to Move Towards a Personalized Evaluation of Psychological Treatments. Canadian Psychology, 53(2), 115-121.

Sales, C., Goncalves, S., Fragoeiro, A., Noronha, S., \& Elliott, R. (2007). Psychotherapists Openness to Routine Naturalistic Idiographic Research. Mental Health and Learning Disabilities Research and Practice, $145-161$.

Soares, L., Cruz, A. S., Moita, G., Sales, C. M. D. \& Alves, P. C. G. (2012, October). Feedback loops between researchers, therapists and patients using IPPS [PowerPoint slides]. Paper presented at the $3^{\text {rd }}$ joint meeting of the Society for Psychotherapy Research European and UK chapters, Porto, Portugal.

Wanigaratne, S., Davis, P., Pryce, K., Brotchie, J. (2005). The effectiveness of psychological therapies on drug misusing clients. London. 
